\title{
Fluorescence of molecules placed near a spherical particle: Rabi splitting
}

\author{
M.M. Dvoynenko \\ V. Lashkaryov Institute of Semiconductor Physics, NAS of Ukraine, \\ 41, prospect Nauky, 03680 Kyiv, Ukraine \\ \& Institute of Atomic and Molecular Sciences, Academia Sinica, Taipei, Taiwan \\ E-mail:mydvoyn@yahoo.com
}

\begin{abstract}
Theoretical study of spontaneously emitted spectra of point-like source placed near spherical Ag particle was performed. It was shown that near-field electromagnetic interaction between a point-like emitter and spherical Ag particle leads to strong coupling between them at very small emitter-metal surface distances. It was shown that values of Rabi splitting are quantitatively close to that of emitter-flat substrate interaction.
\end{abstract}

Keywords: strong coupling, surface plasmon, Rabi splitting.

Manuscript received 29.09.17; revised version received 30.10.17; accepted for publication 07.12.17; published online 07.12.17.

\section{Introduction}

Mechanism of interaction of emitters with surrounding media is a fundamental problem in physics, because it demonstrates that radiation parameters depend not only on an inner structure of the emitter but also on its neighbours. This interaction leads to modification of radiated intensity, emitter lifetime, resonance frequency (Lamb shift) and even Rabi splitting in emitted spectra. Modification of the emitter lifetime (or decay rate) and Lamb shift were studied theoretically [1, 2] and experimentally [3, 4] much more often than strong coupling between emitter and surface plasmon. The extended reviews $[1,2]$ use the classical electrodynamics consideration of the influence of flat substrate on the lifetime and resonance frequency, but the first one [1] describes it in the framework of the local response of the metal substrate, while the second one [2] pays attention to the nonlocal response. Quantum electrodynamics approach was developed and applied in [5] to find the molecule spontaneous emission decay near a flat metal substrate. Dependences of these two parameters on the molecule-metal distance were also studied for a single sphere with local [6-8] and nonlocal [9-12] response of the metal. Dramatic changes in emission spectra, namely, Rabi splitting, of a single emitter placed near a metal structure were not studied in detail, as it was done for the decay rate. There are few publications [13-16] where this problem was theoretically investigated. Different quantum mechanical approaches were applied to study the strong coupling between a molecule and a single spheroidal particle $[13,14]$. The classical electrodynamics approach was applied to study emitted spectra of a quantum dot placed at a dimmer nanoantenna [15] and molecule near flat surface [16]. The influence of the nonlocal response of the metals on emitted spectra was not considered in [13-15]. It is not clear from the mentioned papers what is the reason for Rabi splitting - near-field or far-field: there is some impression that Rabi splitting is associated with far-field. The aim of this paper is to study the influence of many parameters (dipole moment, distance behaviour, etc.) including the metal nonlocal response on the emitted spectra. A comparison of the emitted spectra of a molecule placed near a spherical particle with the case of a flat surface [16] will be made too. 


\section{Fluorescence of an emitter near metal}

As it was written above, there two approaches for the study of interaction of radiating molecules with metal particles: quantum and classical. The spontaneous emitting spectrum of a molecule placed near a metal nanoparticle has been theoretically investigated with the use of the both quantum [14] and classical [16, 17] approaches. This work follows the classical approach. Let us first consider a spontaneous radiating molecule modelled as a dipole - in vacuum undertaking a harmonic oscillation along $z$ axis. The electromagnetic wave emitted from this molecular dipole is scattered by a metal object; the scattered wave then acts on the oscillator. The equation of motion of the molecular dipole placed near the metal object can be written as

$\frac{d^{2} z}{d t^{2}}+\gamma_{0} \frac{d z}{d t}+\omega_{0}^{2} z=\frac{e^{*} E^{r e f}(\vec{r}, t)}{m^{*}}$,

where $m^{*}$ is the effective mass of the dipole, $\gamma_{0}-$ its radiation rate in the vacuum, $\omega_{0}-$ its oscillation frequency, $e^{*}$ - effective electric charge of the dipole, and $E^{r e f}(\vec{r}, t)$ - amplitude of the electric field reflected from the metal object. Note that $\gamma_{0}$ can be interpreted by the action of the dipole on itself [18]. Looking for the solution of Eq. (1) in the form $z=z_{0}(\omega) e^{-i \omega t}$ and consequently for $E^{r e f}(\vec{r}, t)=E^{r e f}(\vec{r}, \omega) e^{-i \omega t}$, Eq. (1) becomes

$$
-\omega^{2} z_{0}-i \gamma_{0} \omega z_{0}+\omega_{0}^{2} z_{0}=\frac{e^{*} E^{r e f}(\vec{r}, \omega)}{m^{*}} .
$$

Since the total emission field is proportional to the dipole $e^{*} z_{0}$, and Eq. (2) is the equation for the eigenfrequency $\omega$, the emitted spectra can be found. The reflected field is defined by

$$
E^{r e f}(\vec{r}, \omega)=k_{0}^{2} / \varepsilon_{0} G_{Z Z}^{r e f}(\omega, \vec{r}, \vec{r}) e^{*} z_{0},
$$

where $k_{0}$ is the wavevector in vacuum, $\varepsilon_{0}$ - vacuum permittivity, $G_{Z Z}^{r e f}(\omega, \vec{r}, \vec{r})-z z$ component of the reflected part of the electrodynamic Green tensor [16]. As a result, Eq. (2) can be rewritten as

$$
\left[-\omega^{2} z_{0}-i \gamma_{0} \omega z_{0}+\omega_{0}^{2} z_{0}-\frac{e^{* 2}}{m^{*}} \frac{k_{0}^{2}}{\varepsilon_{0}} G_{z z}^{r e f}(\omega, \vec{r}, \vec{r})\right] z_{0}=0
$$

Since the dipole moment is in inverse proportion to $\left[-\omega^{2}-i \gamma_{0} \omega+\omega_{0}^{2}-\frac{e^{* 2}}{m^{*}} \frac{k_{0}^{2}}{\varepsilon_{0}} G_{z z}^{r e f}(\omega, \vec{r}, \vec{r})\right]$ and $\gamma_{0}=\frac{e^{2} k_{0}^{3}}{6 \pi \varepsilon_{0} m \omega_{0}}[18,19]$, the emitted intensity $I$ can be written as $[14,20]$

$$
I \sim \frac{\sum_{j=x, y, z} \sin ^{2} \alpha_{j}\left|G_{j z}^{0}\left(\omega, \vec{r}_{D}, \vec{r}\right)+G_{j z}^{r e f}\left(\omega, \vec{r}_{D}, \vec{r}\right)\right|^{2}}{\left|-\omega^{2}-i \gamma_{0} \omega+\omega_{0}^{2}-\gamma 6 \pi c G_{z z}^{r e f}(\omega, \vec{r}, \vec{r})\right|^{2}},
$$

where $G_{j z}^{0}\left(\omega, \vec{r}_{D}, \vec{r}\right)$ is the vacuum electrodynamic Green tensor, $\vec{r}_{D}$ - position of the detector in far-field zone, $\alpha_{j}$-angle between the $j$-axis and $\vec{r}_{D}$. Both the vacuum $G_{j z}^{0}\left(\omega, \vec{r}_{D}, \vec{r}\right)$ and reflected $G_{j z}^{r e f}\left(\omega, \vec{r}_{D}, \vec{r}\right)$ parts of the Green tensor are responsible for the transformation from the near-field zone to the far-field zone. The spectrum depends mainly on the denominator, but is slightly modified by the nominator.

Equations (4) and (5) can be analyzed directly. First, note that the emission spectrum can be modified due to the backscattering field (generalized radiation reaction force). The emitted spectrum is governed by the spectral behavior of $G_{Z Z}^{r e f}(\omega, \vec{r}, \vec{r})$. If the reflected part of the electrodynamic Green's function has no resonance near the eigen-frequency of the molecule in vacuum $\omega_{0}$, one can conclude that the real part of $G_{Z Z}^{r e f}(\omega, \vec{r}, \vec{r})$ is responsible for the Lamb shift: $\Delta \omega \approx \frac{\gamma_{0} 6 \pi c}{2 \omega_{0}} \operatorname{Re}\left[G_{z z}^{r e f}\left(\omega_{0}, \vec{r}, \vec{r}\right)\right]$, while its imaginary part alters the decay rate: $\gamma \approx \gamma_{0}+\frac{\gamma_{0} 6 \pi c}{\omega_{0}} \operatorname{Im}\left[G_{z z}^{r e f}\left(\omega_{0}, \vec{r}, \vec{r}\right)\right][18]$. These effects have been manifested by the huge decay rate for a fluorescent molecule placed near a metal surface $[1,21]$. A spectral shift [21] of molecular fluorescence near Ag surface was displayed too, however, it should be noted the other possible for the shift, namely, molecular aggregations. The decrease of the molecule fluorescence intensity, caused by the energy transfer to the metal surface, leads to a change of the ratio between Raman and fluorescence intensities [22] and was proposed to extract the enhancement factors of SERS [23] and to determine the fluorescence time [24] and molecule-metal distance $[25,26]$. If the resonance frequency $\omega_{r}$ of $G_{z z}^{r e f}(\omega, \vec{r}, \vec{r})$ is near $\omega_{0}$, Eq. (4) can be considered as an equation of two coupled oscillators and can lead to Rabi splitting of the emission spectra [16]. Namely, this case will be considered in this study.

Let us examine the spectral behavior in the case of a spherical particle with the radius $a$ and the dielectric permittivity $\varepsilon_{2}$. Let the molecule dipole is placed in the medium with the dielectric function $\varepsilon_{1}$ near sphere on the axis $O z$ and the distance $r(r>a)$ from the center of the 
sphere. The reflected part of the Green dyadic at the local response of the metal is [27]

$$
\begin{aligned}
& \vec{G}^{r e f}\left(\vec{r}, \vec{r}^{\prime}\right)=\frac{i k_{1}}{4 \pi} \sum_{e} \sum_{o}^{\infty} \sum_{l=1}^{l} \frac{2 l+1}{l(l+1)} \frac{(l-m) !}{(l+m) !}\left(2-\delta_{0 m}\right) \times \\
& {\left[B_{l}^{M} \vec{M}_{{ }_{o} l m}^{(1)}(\vec{r}, k) \otimes \vec{M}_{e_{o} l m}^{(1)}(\vec{r}, k)+B_{l}^{N} \vec{N}_{{ }_{o}}^{(1)}(\vec{r}, k) \otimes \vec{N}_{e_{o} l m}^{(1)}(\vec{r}, k)\right]}
\end{aligned}
$$

where

$$
\begin{aligned}
& \vec{M}_{e_{l} l m}^{(1)}(\vec{r})=\mp \frac{m}{\sin \theta} h_{l}^{(1)}\left(k_{1} r\right) P_{l}^{m}(\cos \theta)\left(\begin{array}{c}
\sin \\
\cos
\end{array}\right)(m \varphi) \vec{e}_{\theta}- \\
& -h_{l}^{(1)}\left(k_{1} r\right) \frac{d P_{l}^{m}(\cos \theta)}{d \theta}\left(\begin{array}{c}
\cos \\
\sin
\end{array}\right)(m \varphi) \vec{e}_{\phi},
\end{aligned}
$$

$$
\begin{aligned}
& \vec{N}_{{ }_{o} l m}^{(1)}(\vec{r})=\frac{l(l+1)}{k_{1} r} h_{l}^{(1)}\left(k_{1} r\right) P_{l}^{m}(\cos \theta)\left(\begin{array}{c}
\cos \\
\sin
\end{array}\right)(m \varphi) \vec{e}_{r}+ \\
& \left.+\frac{1}{k_{1} r} \frac{d\left[r h_{l}^{(1)}\left(k_{1} r\right)\right]}{d r}\right]\left[\frac{d P_{l}^{m}(\cos \theta)}{d \theta}\left(\begin{array}{c}
\cos \\
\sin
\end{array}\right)(m \varphi) \vec{e}_{\theta} \mp\right. \\
& \left.\mp \frac{m}{\sin \theta} P_{l}^{m}(\cos \theta)\left(\begin{array}{l}
\sin \\
\cos
\end{array}\right)(m \varphi) \vec{e}_{\phi}\right]
\end{aligned}
$$

are vector spherical functions, $h_{l}^{(1)}(k r)$ is the spherical Hankel function, $P_{l}^{m}$ is the generalized Legendre function.

$$
B_{l}^{1 M}=-\frac{j_{l}\left(z_{1}\right)\left[z_{2} j_{l}\left(z_{2}\right)\right]^{\prime}-\left[z_{1} j_{l}\left(z_{1}\right)\right]^{\prime} j_{l}\left(z_{2}\right)}{h_{l}^{(1)}\left(z_{1}\right)\left[z_{2} j_{l}\left(z_{2}\right)\right]^{\prime}-\left[z_{1} h_{l}^{(1)}\left(z_{1}\right)\right]^{\prime} j_{l}\left(z_{2}\right)}
$$

and

$$
B_{l}^{1 N}=-\frac{\varepsilon_{2} / \varepsilon_{1} j_{l}\left(z_{2}\right)\left[z_{1} j_{l}\left(z_{1}\right)\right]^{\prime}-j_{l}\left(z_{1}\right)\left[z_{2} j_{l}\left(z_{2}\right)\right]^{\prime}}{\varepsilon_{2} / \varepsilon_{1} j_{l}\left(z_{2}\right)\left[z_{1} h_{l}^{(1)}\left(z_{1}\right)\right]^{\prime}-h_{l}^{(1)}\left(z_{1}\right)\left[z_{2} j_{l}\left(z_{2}\right)\right]^{\prime}}
$$

are reflection coefficients, $z_{i}=k_{i} a$.

In the particle, the $r r$-component of the Green dyadic and for $\theta=0$ is simplified as follows

$$
G_{\perp}^{r e f}(\vec{r}, \vec{r})=\frac{i k_{1}}{4 \pi} \sum_{l=1}^{\infty} l(l+1)(2 l+1) B_{l}^{N}\left(\frac{h_{l}^{(1)}\left(k_{1} r\right)}{k_{1} r}\right)^{2}
$$

while for the dipole parallel to the sphere surface [28]

$$
\begin{aligned}
& G_{\|}^{r e f}(\vec{r}, \vec{r})=\frac{i k_{1}}{4 \pi} \sum_{l=1}^{\infty}(l+1 / 2)\left(B_{l}^{N}\left(\frac{\partial\left(h_{l}^{(1)}\left(k_{1} r\right)\right) / \partial\left(k_{1} r\right)}{k_{1} r}\right)^{2}+\right. \\
& \left.+A_{l}^{N}\left(h_{l}^{(1)}\left(k_{1} r\right)\right)^{2}\right) .
\end{aligned}
$$

When substituting Eqs. (11) and (12) into Eq. (5), one can get spectra of spontaneous emission. In the case of nonlocal response of metals in the hydrodynamic model, the Mie reflection coefficients should be modified [29-31]. Namely, the coefficient $B_{l}^{1 M}$ remains without any changes, while the coefficient $B_{l}^{1 N}$ takes the form [31]

$$
B_{l}^{1 N}=-\frac{\varepsilon_{2} / \varepsilon_{1} j_{l}\left(z_{2}\right)\left[z_{1} j_{l}\left(z_{1}\right)\right]^{\prime}-j_{l}\left(z_{1}\right)\left(\left[z_{2} j_{l}\left(z_{2}\right)\right]^{\prime}+\Delta_{l}\right)}{\varepsilon_{2} / \varepsilon_{1} j_{l}\left(z_{2}\right)\left[z_{1} h_{l}^{(1)}\left(z_{1}\right)\right]^{\prime}-h_{l}^{(1)}\left(z_{1}\right)\left(\left[z_{2} j_{l}\left(z_{2}\right)\right]^{\prime}+\Delta_{l}\right)},
$$

where $\Delta_{l}=l(l+1) j_{l}\left(z_{2}\right) \frac{\varepsilon_{2}-\varepsilon_{\infty}}{\varepsilon_{\infty}} \frac{j_{l}\left(z_{N L}\right)}{z_{N L} j_{l}^{\prime}\left(z_{N L}\right)}, z_{N L}=k_{N L} a$, $\varepsilon_{\infty}$ is a dielectric function of the metal at high frequencies. The longitudinal wavenumber $k_{N L}$ is determined by $k_{N L}^{2}=\left(\frac{\omega_{p}}{\beta_{\mathrm{F}}}\right)^{2} \frac{\varepsilon_{2}}{\varepsilon_{\infty}\left(\varepsilon_{\infty}-\varepsilon_{2}\right)}$, where $\omega_{p}$ is the plasma frequency of the metal, $\beta_{\mathrm{F}}^{2}=\frac{3}{5} \mathrm{v}_{\mathrm{F}}^{2}, \mathrm{v}_{\mathrm{F}}-$ Fermi velocity. Thus, within the hydrodynamic model of the nonlocal response of metals, only the coefficient $B_{l}^{1 N}$ has to be modified, and Eq. (6) may be applied.

\section{Results and discussions}

As we have mentioned, a strong coupling between a single molecule and surface plasmon can occur, if the resonance frequency $\omega_{r}$ of $G_{z z}^{r e f}(\omega, \vec{r}, \vec{r})$ is close to $\omega_{0}$. Resonance properties of $G_{z z}^{r e f}(\omega, \vec{r}, \vec{r})$ can be found from the frequency behaviour of $\operatorname{Im}\left[G_{z z}^{r e f}(\omega, \vec{r}, \vec{r})\right]$, namely, from the behaviour of $\gamma / \gamma_{0}$, since this ratio is much higher than unity for small distances between the molecule and metal surface. The above described theory was applied first of them to a silver sphere, since Rabi splitting was predicted only for $\mathrm{Ag}$ in the case of a flat substrate [16]. The spectral dependence of it on the emitted wavelength is presented in Fig. 1 for local and nonlocal responses of Ag. It is clearly seen that, in the case of the local response of $\mathrm{Ag}$, there is a sharp resonance at the resonance wavelength approximately $340 \mathrm{~nm}$, which corresponds to the real part of the dielectric function of $\mathrm{Ag}[32]$ to be equalled 
to -1 . In other words, this resonance condition is exactly equalled to the one of the case of a small distance between a molecule and flat substrate [16]. Indeed, it has been noted in $[8,33]$ that at very small distances between the dipole and sphere surface as compared to the sphere radius, the sphere can be considered as a flat substrate from the point of view of the reflected part of the Green function. In other words, the well-known electrostatic approximation $[1,20]$ may be applied in this case. The nonlocality of the metal response reduces the $\mathrm{Q}$-factor of the resonance, but its peak is still nearby this wavelength. Thus, we will investigate the spontaneous emitted spectra at the resonance wavelength of the emitter in vacuum to be $340 \mathrm{~nm}$. This wavelength $(340 \mathrm{~nm})$ corresponds to $\omega_{0}$ in all equations above.

The spontaneous emission spectra at different molecule-metal distances, the sphere radius of $25 \mathrm{~nm}$ and radiation rate of $\gamma_{0}=10^{9} \mathrm{~s}^{-1}$ are shown in Fig. 2. It is clearly seen that Rabi splitting is not symmetrical. This asymmetry is associated with the resonance condition of surface plasmon [34]: $\operatorname{Re}\left[\varepsilon_{2}\right]<0$. The wavelength of $320 \mathrm{~nm}$ corresponds to $\operatorname{Re}\left[\varepsilon_{2}\right]=0$. So, the wavelength of $320 \mathrm{~nm}$ looks like a wall. The other feature of Rabi splitting is its dependence on the distance between the emitter and particle surface. As for the flat substrate [16], Rabi splitting can be observed only for small distances. Typical inhomogeneous line broadening of fluorescence is about $10 \mathrm{~nm}$ for this frequency range. So, even for the emitter-metal distances of $1.5 \mathrm{~nm}$ Rabi splitting can be observed according to the theory within local response of metals. However, already at the emitter-metal surface distance of $2 \mathrm{~nm}$, the splitting is not predicted at this response. The position of the upper peak can be a good indicator for the emitter-metal distances.

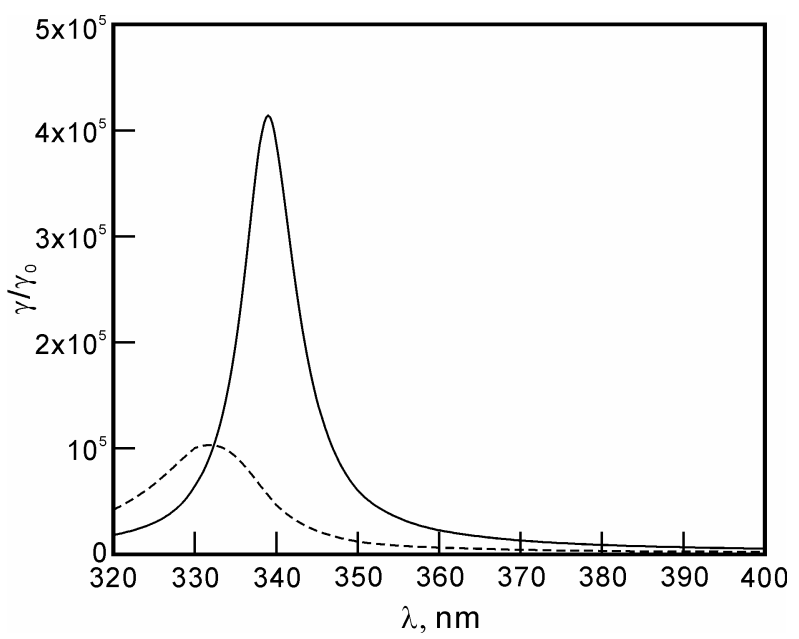

Fig. 1. The spectral dependence of $\gamma / \gamma_{0}$ for the particles with radius $a=25 \mathrm{~nm}$ and the distance from the sphere centre to dipole location $r=26 \mathrm{~nm}$ in local (solid line) and nonlocal (dashed line) responses of Ag.

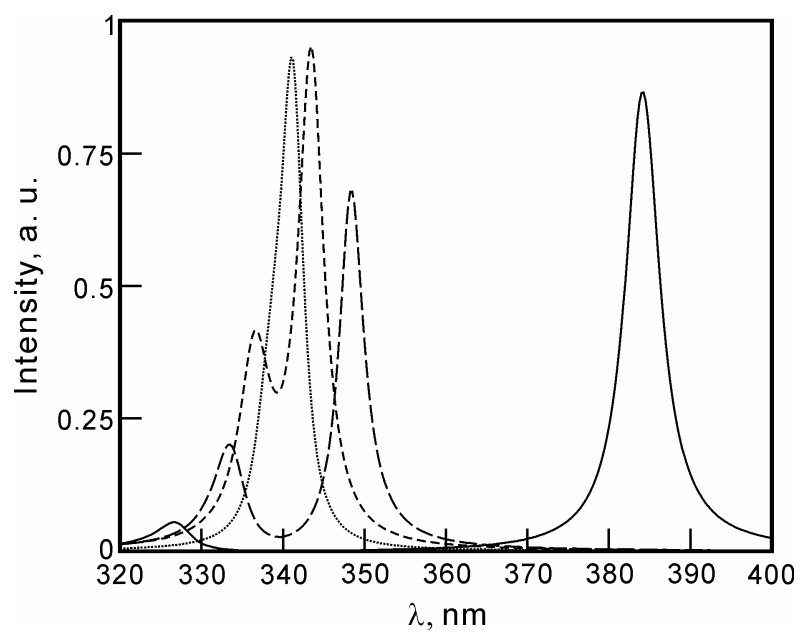

Fig. 2. The fluorescence spectra at $a=25 \mathrm{~nm}$ and $r=25.5 \mathrm{~nm}$ (solid line), $r=26 \mathrm{~nm}$ (dashed line), $r=26.5 \mathrm{~nm}$ (short dashed line) and $r=27 \mathrm{~nm}$ (dotted line).

One more important parameter influencing on the emitting spectra is the dipole of the emitter or its radiation rate $\gamma_{0}$. The higher value of radiation rate leads to the higher value of splitting [16]. The spontaneous emission spectra at different values of $\gamma_{0}$ and at the sphere radius of $25 \mathrm{~nm}$, the molecule-metal distance of $0.5 \mathrm{~nm}$ are shown in Fig. 3. It is seen that the splitting at $\gamma_{0}=10^{9} \mathrm{~s}^{-1}$ is much higher than that at $2 \cdot 10^{8} \mathrm{~s}^{-1}$. In the case of flat substrate, Rabi splitting is roughly proportional to $\sqrt{\gamma_{0}}$. The deviation from this rule is caused by the existence of the loss conditioned by metal absorption [35]. One can check from Fig. 3 that the splitting is not exactly proportional to $\sqrt{\gamma_{0}}$, which can be associated with the mentioned contribution of the losses to Rabi splitting.

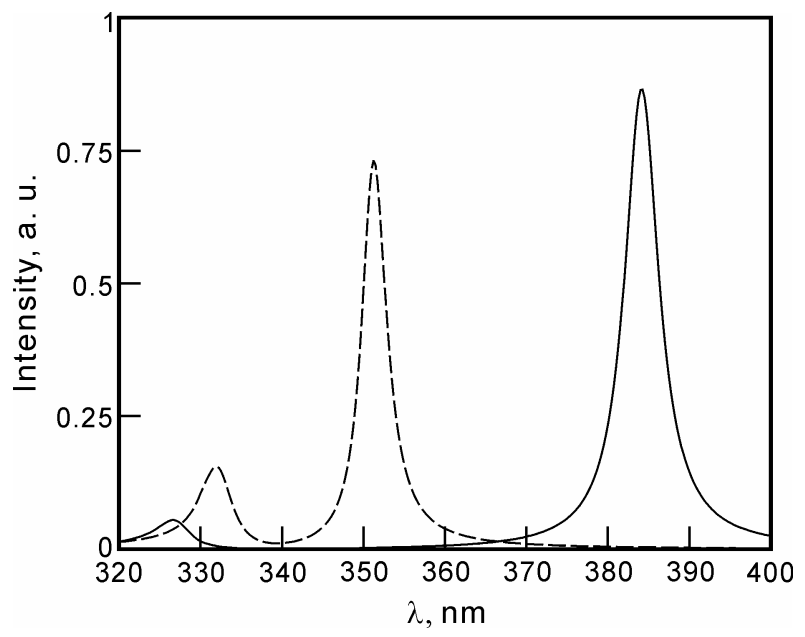

Fig. 3. The fluorescence spectra at $a=25 \mathrm{~nm}, r=25.5 \mathrm{~nm}$ and $\gamma_{0}=10^{9} \mathrm{~s}^{-1}$ (solid line) and $\gamma_{0}=2 \cdot 10^{8} \mathrm{~s}^{-1}$ (dashed line). 
The orientation of the dipole is also important for Rabi splitting, because it influences on the decay rate in the case of a flat substrate [1]. The spontaneous emission spectra at two different values of $\gamma_{0}$, at the sphere radius of $25 \mathrm{~nm}$, the molecule-metal distance of $0.5 \mathrm{~nm}$ and the perpendicular and parallel orientations of the emitting dipole are shown in Fig. 4. Since $\gamma_{0}$ is twice lower in the case of flat substrate for the parallel dipole as compared to the perpendicular one in the electrostatic approximation [1] and the mentioned above of the Rabi splitting dependence on $\gamma_{0}$, one could expect that the splitting should be $\sqrt{2}$ lower for the parallel orientation as compared to the perpendicular one. However, one can check that there is a deviation from this rule, and it is caused by the mentioned contribution of the losses to Rabi splitting.

At the distances between metal particles smaller than few nanometers, the nonlocal response of metals [31] and electron tunnelling effect [36] between the particles become important. Thus, it is expected that, at the distances between emitter and metal surface of $0.5-2 \mathrm{~nm}$, the nonlocality in the metal response is also sensitive. Thus, with the use of the mentioned in previous section hydrodynamical approach, the emitted spectra were calculated with nonlocal response of silver. Needed parameters of $\mathrm{Ag}$ has been taken from Ref. [37]. The calculated data are presented in Fig. 5a. It is seen that nonlocal response of metals reduces the value of Rabi splitting roughly twice. For the comparison, the emitted spect$\mathrm{ra}$ at the same parameters of emitter-metal distances $(z=$ $0.5 \mathrm{~nm}$ ) of flat metal surface [16] are shown in this figure, too. One can find that the deviation between these two cases (emitter at sphere and emitter at flat surface) is not high. The emitted spectra are determined by the distance from metal surface (sphere or flat substrate) to emitter. In this case, the emitter plays a role of a detector, too. However, local field spectra are observed usually at the plane wave excitation. In other words, the emitter is located in far-field zone, while the detector is near the metal particle. Fig. 5b represents the local field enhancement factor $\left(E / E_{0}\right)$ of $\mathrm{Ag}$ sphere with two radiuses of 25 and $5 \mathrm{~nm}$ at the distance between the observation point and the sphere surface to be $0.05 \mathrm{~nm}$ and in the direction of applied electric field. Here, $E$ is the amplitude of the field in the mentioned point, and $E_{0}$ is the amplitude of the incident plane wave. Fig. $5 \mathrm{~b}$ clearly shows that there is a small red shift in the local field enhancement factor at the nonlocal response as compared to the local one [38]. On the other hand, Rabi splitting at the nonlocal response is roughly reduced twice (Fig. 5a) as compared to the local one.

The emitted spectra have been tested by different size of the spheres. Fig. 6 shows the emitted spectra for the two values of the radiuses, namely 25 and $5 \mathrm{~nm}$. One can find that the splitting almost does not depend on the sphere radius. It was not expected, because according to quantum electrodynamics consideration $[39,40]$ lightmatter interaction is inversely proportional to square root of effective volume. The effective volume for the sphere with smaller size is expected to be lower. This problem is needed for a detail and separate consideration.

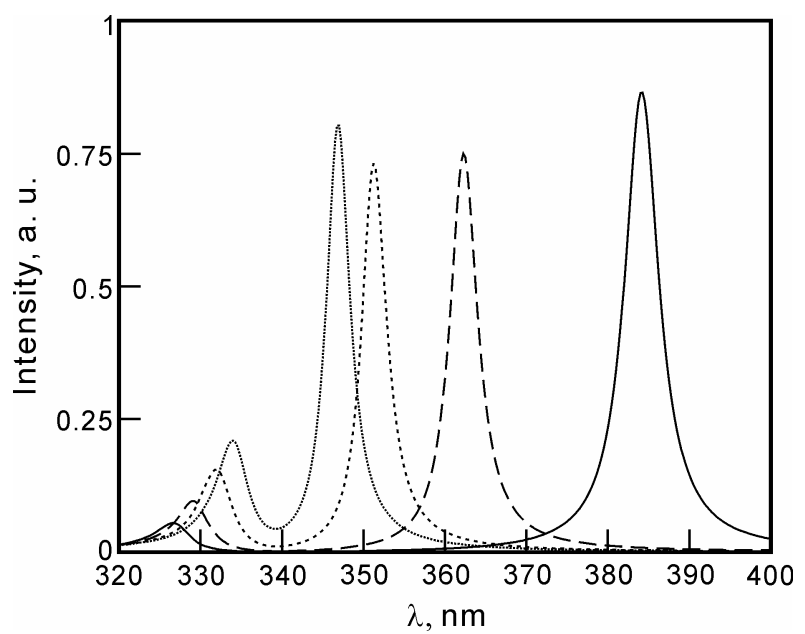

Fig. 4. The fluorescence spectra for $a=25 \mathrm{~nm}, r=25.5 \mathrm{~nm}$ at $\gamma_{0}=10^{9} \mathrm{~s}^{-1}$ (solid and dashed lines) and $2 \cdot 10^{8} \mathrm{~s}^{-1}$ (short dashed and dotted lines). The orientation of the dipole is perpendicular (solid and short dashed lines) to the surface and parallel (dashed and dotted lines) to it.
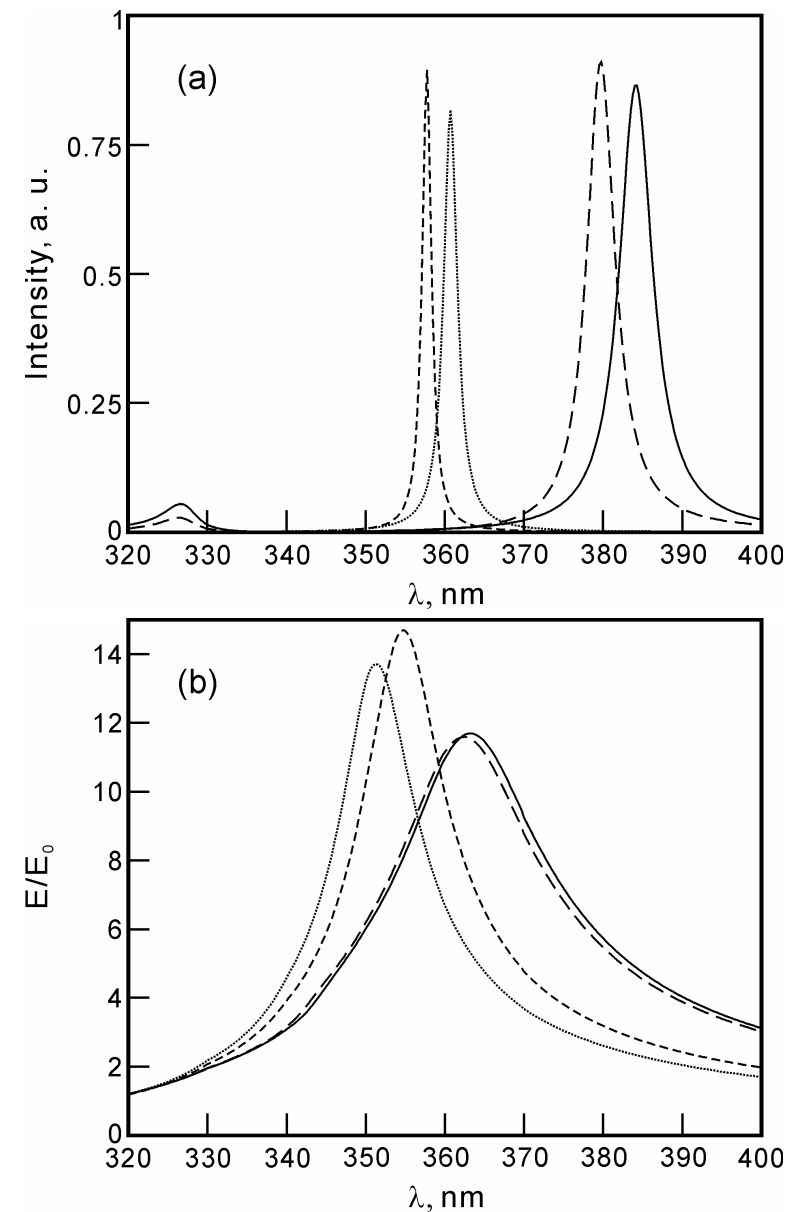

Fig. 5. (a) The fluorescence spectra for $a=25 \mathrm{~nm}, r=25.5 \mathrm{~nm}$ (solid and short dashed lines) and $z=0.5 \mathrm{~nm}$ (dashed and dotted lines) $\mathrm{nm}$ at the local (solid and dashed lines) and nonlocal (short dashed and dotted lines) responses of Ag. (b) The spectral dependence of the local field enhancement factor $\left(E / E_{0}\right)$ for $a=25 \mathrm{~nm}$ (solid and dashed lines) and $a=5 \mathrm{~nm}$ (short dashed and dotted lines) at the local (solid and dashed lines) and nonlocal (short dashed and dotted lines) responses of Ag. 


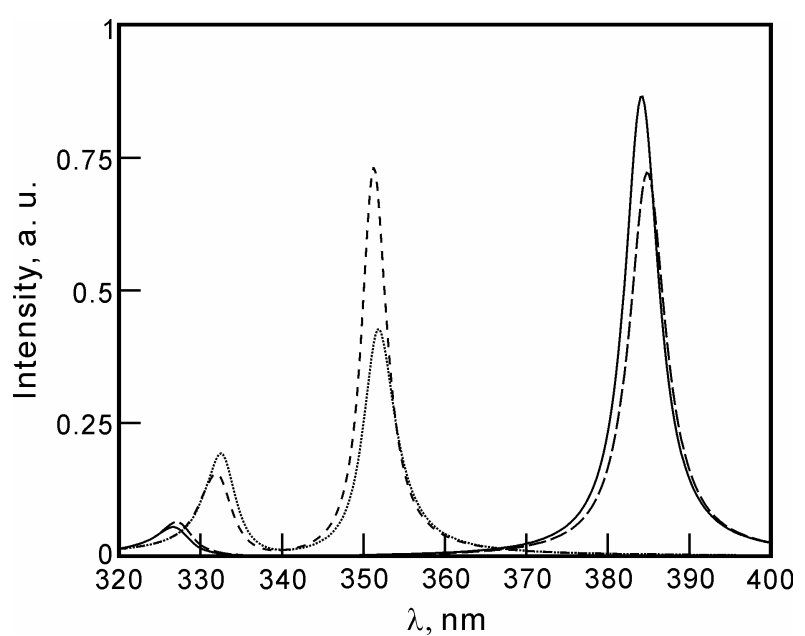

Fig. 6. The fluorescence spectra at $a=25 \mathrm{~nm}, r=25.5 \mathrm{~nm}$ (solid and short dash) and $a=5 \mathrm{~nm}, r=5.5 \mathrm{~nm}$ (dash and dot) for $\gamma_{0}=10^{9} \mathrm{~s}^{-1}$ (solid and dash) and $2 \cdot 10^{8} \mathrm{~s}^{-1}$ (short dash and dot).

\section{Conclusions}

The problem of strong coupling in light-matter interaction of a point-like source placed near a spherical silver particle was considered using the classical electrodynamics way. The strong coupling is manifested in spontaneous emission spectra. Particularly, Rabi splitting can occur at some special conditions. The main behaviours of this phenomenon are as follows:

- Rabi splitting can be resolved at very small distances between the emitter and particle surface (up to $1 \ldots 1.5 \mathrm{~nm}$ );

- the dipole orientation and its radiation rate affect on the splitting;

- near Ag surface, the resonance of backscattering waves occurs at about $340 \mathrm{~nm}$, where the real part of the dielectric permittivity of Ag equals to - 1 , but not at the wavelength having resonance in far-field zone;

- nonlocal response of metals reduces Rabi splitting.

The study also showed that all behaviours mentioned above are similar to the case of the point-like source placed near a flat Ag substrate (see also Ref. [16]).

\section{References}

1. Chance R.R., Prock A., and Silbey R. Molecular fluorescence and energy transfer near interfaces. Adv. Chem. Phys. 1978. 37. P. 1-65.

2. Ford G.W. and Weber W.H. Electromagnetic interactions of molecules with metal surfaces. Phys. Rep. 1984. 113. P. 195-287.

3. Drexhage K.H. Influence of a dielectric interface on fluorescence decay. J. Lumin. 1970. 1, 2. P. 693-701.
4. Cnossen G., Drabe K.E. and Wiersma D.A. Fluorescence properties of submonolayers of rhodamine $6 \mathrm{G}$ in front of a mirror. J. Chem. Phys. 1993. 98. P. 5276-5280.

5. Pipa V.I. Electromagnetic field quantization in planar absorbing heterostructures. SPQEO. 2011. 14. P. 91-97.

6. Ruppin R. Decay of an excited molecule near a small metal sphere. J. Chem Phys. 1982. 76. P. 1681-1684.

7. Chew H. Transition rates of atoms near spherical surfaces. J. Chem. Phys. 1987. 87. P. 1355-1360.

8. Dung H.T., Knoll L., and Welsch D.-G. Decay of an excited atom near an absorbing microsphere. Phys. Rev. A. 2001. 64. P. 013804.

9. Vielma J. and Leung P.T. Nonlocal optical effects on the fluorescence and decay rates for admolecules at a metallic nanoparticle. J. Chem. Phys. 2007. 126. P. 194704.

10. Leung P.T. Decay of molecules at spherical surfaces: Nonlocal effects. Phys. Rev. B. 1990. 42. P. 7622-7625.

11. Castanie E., Boffety M., and Carminati R. Fluorescence quenching by a metal nanoparticle in the extreme near-field regime. Opt. Lett. 2010. 35. P. 291-293.

12. Ruppin R. Optical properties of small spheres. Phys. Rev. B. 1975. 11. P. 2871-2876.

13. Trugler A. and Hohenester U. Strong coupling between a metallic nanoparticle and a single molecule. Phys. Rev. B. 2008. 77. P. 115403.

14. Van Vlack C., Kristensen P.T., and Hughes S. Spontaneous emission spectra and quantum lightmatter interactions from a strongly coupled quantum dot metal-nanoparticle system. Phys. Rev. B. 2012. 85. P. 075303.

15. Savasta S., Saija R., Ridolfo A., Di Stefano O., Denti P., and Borghese F. Nanopolaritons: Vacuum Rabi splitting with a single quantum dot in the center of a dimer nanoantenna. ACS Nano. 2010. 4. P. 6369-6376.

16. Dvoynenko M.M. and Wang J.-K. Revisiting strong coupling between a single molecule and surface plasmons. Opt. Lett. 2013. 38. P. 760-762.

17. Childs J.J., An K., Dasari R.R., and Feld M.S. Single atom emission in an optical resonator, Chap. 8 in: Cavity Quantum Electrodynamics, ed. P.R. Barman, pp. 325-379, Academic Press, Boston, 1994.

18. Hinds E.A. Perturbative Cavity Electrodynamics, Chap. 1 in: Cavity Quantum Electrodynamics, ed. P.R. Berman. Academic Press, Boston, 1994. P. 156.

19. Feynman R., Leighton R. and Sachs M. The Feynman Lectures on Physics, vol. I. AddisonWesley, 1977. P. 32-1.

20. Dvoynenko M.M. and Wang J.-K. Can electrodynamic interaction between amolecule and metal dominate a continuum background in surface- 
enhanced Raman scattering? Phys. Chem. Chem. Phys. 2015. 17. P. 27258-27263.

21. Cnossen G., Drabe K.E., and Wiersma D.A. Fluorescence properties of submonolayers of rhodamine 6Gin front of a mirror. J. Chem. Phys. 1993. 98. P. 5276-5280.

22. Johansson P., Xu H. and Käll M. Surface-enhanced Raman scattering and fluorescence near metal nanoparticles. Phys. Rev. B. 2005. 72. P. 035427.

23. Dvoynenko M.M. and Wang J.-K. Finding electromagnetic and chemical enhancement factors of surface-enhanced Raman scattering. Opt. Lett. 2007. 32. P. 3552-3554.

24. Galloway C.M., Etchegoin P.G. and Le Ru E.C. Ultrafast nonradiative decay rates on metallic surfaces by comparing surface-enhanced Raman and fluorescence signals of single molecules. Phys. Rev. Lett. 2009. 103. P. 063003.

25. Dvoynenko M.M., Kazantseva Z.I., Strelchuk V.V., Kolomys O.F., Venger E.F. and Wang J.-K. Molecular ruler based on concurrent measurements of enhanced Raman scattering and fluorescence. Opt. Lett. 2010. 35. P. 3808-3810.

26. Dvoynenko M.M., Kazantseva Z.I., Strelchuk V.V., Kolomys O.F., Bortshagovsky E.G., Venger E.F., Tronc P. Probing plasmonic system by the simultaneous measurement of Raman and fluorescence signals of dye molecules. SPQEO. 2011. 14. P. 195-199.

27. Li L.-W., Kooi P.-S., Leong M.-S., Yee T.-S. Electromagnetic dyadic Green's function in spherically multilayered media. IEEE Trans. Microwave. 1994. 42. P. 2302-2310.

28. Kim Y.S., Leung P.T. and George T.F. Classical decay rates for molecules in the presence of a spherical surface: a complete treatment. Surf. Sci. 1988. 195. P. 1-14.

29. Ruppin R. Optical properties of a plasma sphere. Phys. Rev. Lett. 1973. 31. P. 1434-1437.

30. Datsyuk V.V. A generalization of the Mie theory for a sphere with spatially dispersive permittivity. Ukr. J. Phys. 2011. 56. P. 122-129.
31. Christensen T., Yan W., Raza S., Jauho A.-P., Mortensen N.A., and Wubs M. Nonlocal response of metallic nanospheres probed by light, electrons, and atoms. ACS Nano. 2014. 8. P. 1745-1758.

32. Yang H.U., D'Archangel J., Sundheimer M.L., Tucker E., Boreman G.D., and Raschke M.B. Optical dielectric function of silver. Phys. Rev. B. 2015. 91. P. 235137.

33. Colas des Francs G., Bouhelier A., Finot E., Weeber J.C., Dereux A., Girard C. and Dujardin E. Fluorescence relaxation in the near-field of a mesoscopic metallic particle: distance dependence and role of plasmon modes. Opt. Exp. 2008. 16. P. 17654-17666.

34. Stockman M.I. Nanoplasmonics: Past, present, and glimpse into future. Opt. Exp. 2011. 19. P. 22029 22106.

35. Dvoynenko M.M. and Wang J.-K. Rabi splitting at intersubband transition assisted by longitudinal optical phonon. Phys. Rev. B. 2007. 75. P. 245315.

36. Zhu W., Esteban R., Borisov A.G., Baumberg J.J., Nordlander P., Lezec H.J., Airpurua J. and Grozier K.B. Quantum mechanical effects in plasmonic structures with subnanometre gaps. Nat. Commun. 2016. 7. P. 11495.

37. Klimov V. Nanoplasmonics. CRC Press, Boca Raton, 2013.

38. C. David and F.J. García de Abajo, Spatial Nonlocality in the Optical Response of Metal Nanoparticles. J. Phys. Chem. C 115, pp. 19470 19475 (2011).

39. Fox M. Quantum Optics. An Introduction. Oxford University Press, Oxford, 2006.

40. Chikkaraddy R., de Nijs B., Benz F., Barrow S.J., Scherman O.A., Rosta E., Demetriadou A., Fox P., Hess O. and Baumberg J.J. Single-molecule strong coupling at room temperature in plasmonic nanocavities. Nature. 2016. 535. P. 127-130. 